\section{OPEN ACCESS}

Edited by:

Xiaojuan $\mathrm{He}$,

China Academy of Chinese Medical Sciences, China

*Correspondence:

Hao Xu

hoxu@163.com

Qianqian Liang

liangqianqiantcm@126.com

${ }^{\dagger}$ These authors have contributed equally to this work

Specialty section:

This article was submitted to

Ethnopharmacology,

a section of the journal

Frontiers in Pharmacology

Received: 10 May 2020 Accepted: 10 July 2020

Published: 24 July 2020

Citation:

Wang $T$, Jia $Q$, Chen $T$, Yin $H$, Tian $X$, Lin $X$, Liu $Y$, Zhao $Y$, Wang $Y$, Shi $Q$, Huang C, Xu H and Liang Q (2020) Corrigendum: Alleviation of Synovial Inflammation of Juanbi-Tang on Collagen-Induced Arthritis and TNF-Tg Mice Model.

Front. Pharmacol. 11:1127. doi: 10.3389/fphar.2020.01127

\title{
Corrigendum: Alleviation of Synovial Inflammation of Juanbi-Tang on Collagen-Induced Arthritis and TNF-Tg Mice Model
}

\begin{abstract}
Tengteng Wang ${ }^{1,2 \dagger}$, Qingyun $\mathrm{Jia}^{3 \dagger}$, Tao Chen ${ }^{1,2,4 \dagger}$, Hao Yin ${ }^{5}$, Xiaoting Tian ${ }^{5}$, Xi Lin $^{6}$, Yang Liu ${ }^{1,2,4}$, Yongjian Zhao ${ }^{1,2,4}$, Yongjun Wang ${ }^{1,2,4}$, Qi Shi ${ }^{1,2,4}$, Chenggang Huang ${ }^{5}$, Hao $\mathrm{Xu}^{1,2,4 *}$ and Qianqian Liang ${ }^{1,2,4 *}$

${ }^{1}$ Longhua Hospital, Shanghai University of Traditional Chinese Medicine, Shanghai, China, ${ }^{2}$ Institute of Spine, Shanghai University of Traditional Chinese Medicine, Shanghai, China, ${ }^{3}$ Second Ward of Trauma Surgery Department, Linyi People's Hospital, Linyi, China, ${ }^{4}$ Key Laboratory of Theory and Therapy of Muscles and Bones, Ministry of Education, Shanghai University of Traditional Chinese Medicine, Shanghai, China, ${ }^{5}$ Shanghai Institute of Materia Medica, Chinese Academy of Sciences, Shanghai, China, ${ }^{6}$ Department of Pathology and Laboratory Medicine, University of Rochester, Rochester, NY, United States
\end{abstract}

Keywords: Juanbi-Tang, rheumatoid arthritis, collagen-induced arthritis, synoviocyte, tumor necrosis factor

\section{A Corrigendum on}

Alleviation of Synovial Inflammation of Juanbi-Tang on Collagen-Induced Arthritis and TNFTg Mice Model

By Wang T, Jia Q, Chen T, Yin H, Tian X, Lin X, Liu Y, Zhao Y, Wang Y, Shi Q, Huang C, Xu H and Liang Q (2020) Alleviation of Synovial Inflammation of Juanbi-Tang on Collagen-Induced Arthritis and TNF-Tg Mice Model. Front. Pharmacol. 11:45. doi: 10.3389/fphar.2020.00045

The authors found that Figure 2F was incorrect. During the first revision, the authors replaced the bar graph with a dot plot according to a reviewer's suggestion; however, the authors missed Figure 2F and placed Figure 2D where Figure 2F should be placed. The correct Figure 2F appears below.

The authors apologize for this error and state that this does not change the scientific conclusions of the article in any way. The original article has been updated.

\footnotetext{
Copyright () 2020 Wang, Jia, Chen, Yin, Tian, Lin, Liu, Zhao, Wang, Shi, Huang, Xu and Liang. This is an open-access article distributed under the terms of the Creative Commons Attribution License (CC BY). The use, distribution or reproduction in other forums is permitted, provided the original author $(s)$ and the copyright owner(s) are credited and that the original publication in this journal is cited, in accordance with accepted academic practice. No use, distribution or reproduction is permitted which does not comply with these terms.
} 


\section{A}

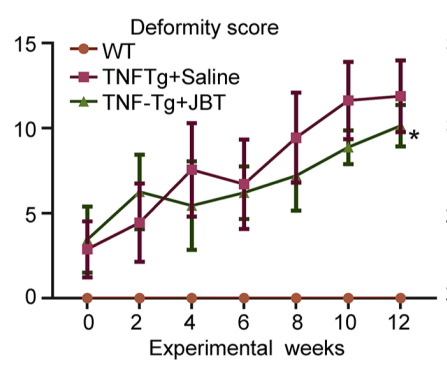

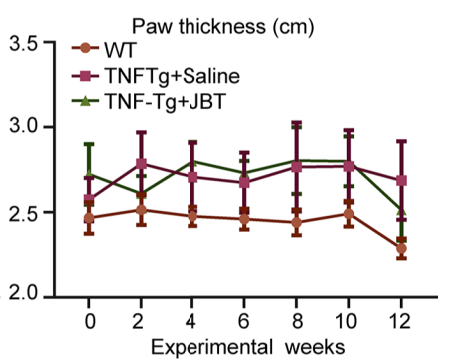

TNF-Tg

C

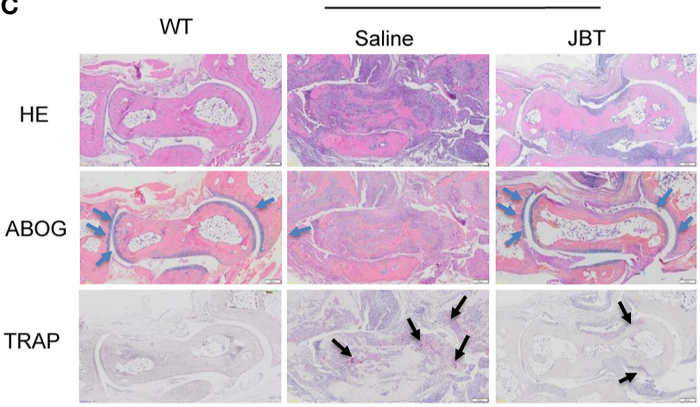

D

Inflammatory synovial area $\left(\mathrm{mm}^{2}\right)$

E
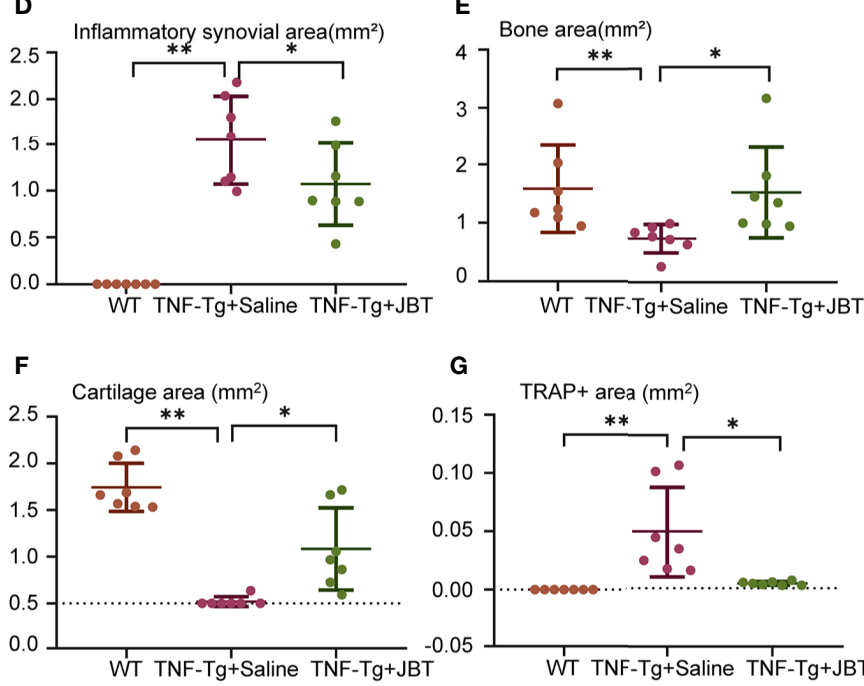

G

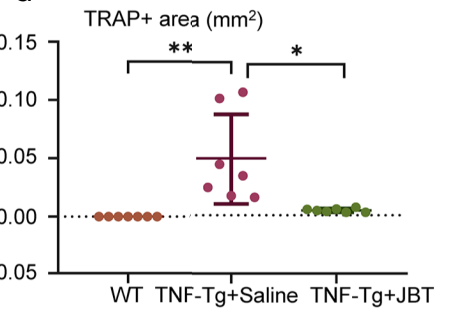

H

WT

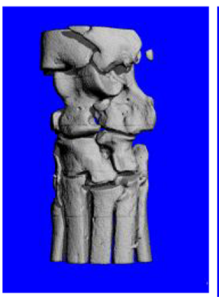

TNF-Tg

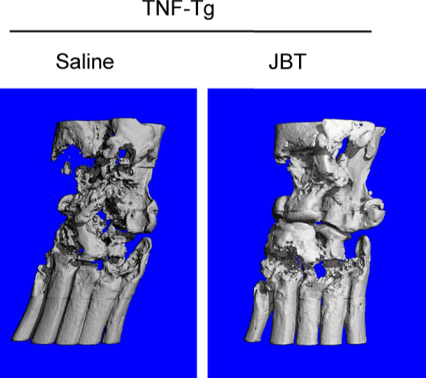

FIGURE 2 | Juanbi-Tang (JBT) attenuated symptoms, inflammatory synovial volume and bone erosion at ankle joint of tumor necrosis factor transgenic (TNF-Tg) mice. The deformity score (A) and hind paw thickness (B) of TNF-Tg mice and WT littermates were scored and recorded every week in a blinded manner.

(C) Hematoxylin-eosin staining, ABOG staining, and TRAP staining were used to analyze the ankle joints of WT and TNF-Tg mice, which were treated with saline or JBT for 12 weeks. Bar, $200 \mu \mathrm{m}$. (D) Histomorphometric assessment of inflammatory synovial volume area. (E) Histomorphometric assessment of bone area.

(F) Histomorphometric assessment of cartilage area. (G) Histomorphometric assessment of TRAP+ osteoclast area. (H) Micro-CT of ankle joints. Results are shown as mean \pm SEM, for 6-8 legs per group. Three sections per mice were analyzed. ${ }^{* *} \mathrm{p}<0.01$ versus TNF-Tg + saline, ${ }^{\star} \mathrm{p}<0.05$ versus TNF-Tg + saline. 